\title{
Beam energy distribution influences on density modulation efficiency in seeded free-electron lasers
}

\author{
Guanglei Wang, Weiqing Zhang, Guorong Wu, Dongxu Dai, and Xueming Yang* \\ State Key Laboratory of Molecular Reaction Dynamics, Dalian Institute of Chemical Physics, \\ Chinese Academy of Sciences, Dalian 116023, People's Republic of China
}

Chao Feng, Meng Zhang, Haixiao Deng, ${ }^{\dagger}$ Dong Wang, and Zhentang Zhao

Shanghai Institute of Applied Physics, Chinese Academy of Sciences, Shanghai 201800, People's Republic of China

(Received 31 December 2014; published 4 June 2015)

\begin{abstract}
The beam energy spread at the entrance of an undulator system is of paramount importance for efficient density modulation in high-gain seeded free-electron lasers (FELs). In this paper, the dependences of high harmonic bunching efficiency in high-gain harmonic generation (HGHG), echo-enabled harmonic generation (EEHG) and phase-merging enhanced harmonic generation (PEHG) schemes on the electron beam energy spread distribution are studied. Theoretical investigations and multidimensional numerical simulations are applied to the cases of uniform and saddle beam energy distributions and compared to a traditional Gaussian distribution. It shows that the uniform and saddle electron energy distributions significantly enhance the bunching performance of HGHG FELs, while they almost have no influence on EEHG and PEHG schemes. A further start-to-end simulation example demonstrated that, with the saddle distribution of sliced beam energy spread controlled by a laser heater, the 30th harmonic can be directly generated by a single-stage HGHG scheme for a soft x-ray FEL facility.
\end{abstract}

DOI: 10.1103/PhysRevSTAB.18.060701

PACS numbers: $41.60 . \mathrm{Cr}$

\section{INTRODUCTION}

In recent years, enormous progress has been achieved in the seeded free-electron lasers (FELs), which hold great potential to deliver high brilliance radiation pulses with excellent longitudinal coherence in the extreme ultraviolet and even x-ray regions. The first seeding scheme, i.e., highgain harmonic generation (HGHG) has been fully demonstrated at BNL [1-4] and is currently used to deliver coherent extreme ultraviolet FEL pulses to users at FERMI [5]. For a long time, it is thought that the frequency multiplication factor of a single-stage HGHG is usually limited within $\sim 10[1,6]$, due to the tradeoff between the energy modulation and the energy spread requirement for exponential amplification process of FEL. Therefore, a complicated multistage HGHG scheme [7-9] has been theoretically proposed and experimentally demonstrated for short wavelength production from a commercially available seed laser.

\footnotetext{
* Corresponding author. xmyang@dicp.ac.cn

Corresponding author. denghaixiao@sinap.ac.cn

Published by the American Physical Society under the terms of the Creative Commons Attribution 3.0 License. Further distribution of this work must maintain attribution to the author $(s)$ and the published article's title, journal citation, and DOI.
}

Meanwhile, alternative seeding concepts are under investigation to enhance the frequency up-conversion efficiency. The well-known echo-enabled harmonic generation (EEHG) scheme with a dual modulator-chicane system has the potential to work efficiently at several tens of harmonic number in a single-stage configuration [10-11]. Recent efforts have demonstrated that the 3rd, 4th, 7th and 15th harmonics of the seed laser could be generated by a single-stage EEHG [11-15]. More recently, another advanced seeding scheme termed phase-merging enhanced harmonic generation (PEHG) has been proposed [16-18], which benefits from the transverse-longitudinal coupling of the electron beam phase space. The novel design of PEHG equivalently suppresses the beam energy spread and future demonstrates remarkable harmonic upconversion efficiency in a single stage. Currently, a proofof-principle PEHG experiment [19] is under way at Shanghai deep ultraviolet FEL test facility [20-22].

Up to now, the bunching performance assessment for seeded FELs is on the basis of assumption that the electron beam at the entrance of the undulator has an energy spread of Gaussian distribution, which however is not true, e. g., in the specific case with a laser heater in the LINAC [23-24]. Laser heater is widely utilized in high-gain FEL facilities to suppress the gain of the microbunching instability via Landau damping by controllably increasing the beam energy spread. It is found that a non-Gaussian energy distribution can be induced by a laser heater and inherited 
in the main LINAC section, depending upon details of the transverse overlap between the laser beam and the electron beam in the laser heater system. A recent experiment at FERMI $[5,25]$ demonstrates that the non-Gaussian beam energy spread induced by the laser heater may expand the harmonic number of a single-stage HGHG to several tens [26-27]. Meanwhile, one cannot exclude other unknown schemes lie beyond the horizon for controlling beam energy spread distribution in the future.

Considering that the initial energy distribution of electron beam is one of the most critical elements in the bunching process of seeded FELs, in this paper, the possible beam energy distribution influences on density modulation efficiency in various seeded FEL schemes have been studied. In Sec. II, by using a set of nominal parameters of the Shanghai soft x-ray free-electron laser facility (SXFEL) [28], the bunching efficiencies in HGHG, EEHG and PEHG schemes with different electron beam energy spread distribution are theoretically derived and numerically simulated, which shows that the uniform and saddle cases may significantly enhance the bunching performance of HGHG. It indicates that the beam energy distribution is of great importance for the HGHG scheme, the frequency up-conversion number of a single-stage HGHG can be improved to 30 or even higher with a uniform or saddle electron energy distribution. In contrast, EEHG and PEHG strongly change and reset the longitudinal beam phase space; their performances depend weakly on the initial beam energy distribution. A followed start-to-end example in Sec. III demonstrated that the saddle distribution of sliced beam energy spread controlled by a laser heater can be maintained in the following accelerations of LINAC, and the saddle beam energy distribution is capable of driving a 30th harmonic upconversion in a single-stage HGHG operation of SXFEL, even though it has a larger sliced beam energy spread than a Gaussian case. Finally, we present our conclusions in Sec. IV.

\section{ENERGY SPREAD DISTRIBUTION EFFECTS ON SEEDING SCHEME}

In order to obtain a comprehensible idea of the energy spread distribution effects on different seeded configurations, by using the nominal parameters of SXFEL, uniform and saddle energy spread distributions are investigated for the density modulation process and compared to the previous Gaussian distribution case in this section, under the same rms deviation, i.e., beam energy spread. SXFEL aims at generating coherent $8.8 \mathrm{~nm}$ FEL pulses from $264 \mathrm{~nm}$ seed laser through a two-stage HGHG. In the nominal design of SXFEL, an $840 \mathrm{MeV}$ electron beam with sliced energy spread of $84 \mathrm{keV}$, i.e., a relative energy spread of $1 \times 10^{-4}$, normalized emittance of $1.0 \mu \mathrm{m}-\mathrm{rad}$, bunch charge of $500 \mathrm{pC}$, and peak current of $500 \mathrm{~A}$ is expected at the exit of the LINAC for efficient FEL lasing. The sliced

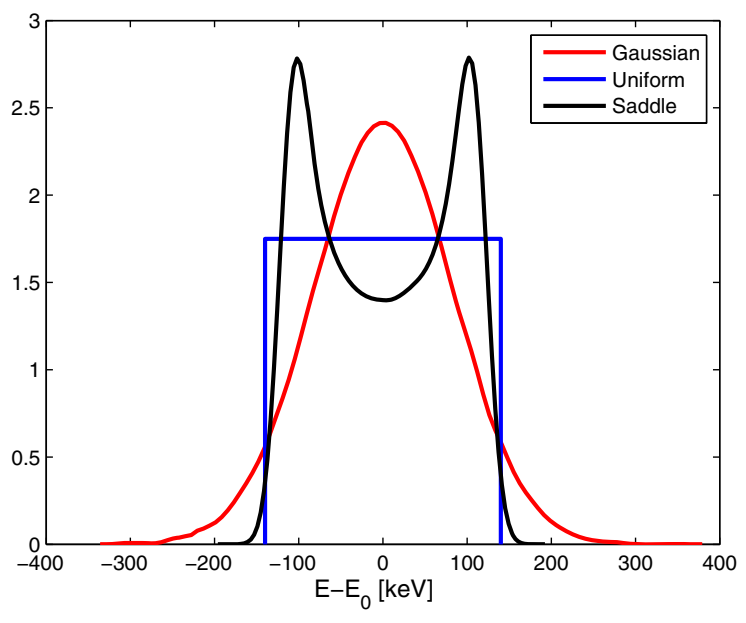

FIG. 1. The different beam energy distributions with rms energy spread of $84 \mathrm{keV}$ for the studies in this section.

beam energy distributions used in the frame of analysis of this section are summarized in Fig. 1.

It is necessary to take some words to describe the saddle distribution before we step forward, while the Gaussian and uniform distributions are quite straight. As is well known, laser heaters used for microbunching instability suppression in modern high-brightness LINACs have shown the possibility to control the rms deviation and the distribution shape of sliced beam energy spread by choosing the laser spot size and the peak power $[23,26]$. In more detail, in the LINAC of SXFEL, electrons from the photoinjector are first accelerated up to $130 \mathrm{MeV}$, and then sent into the laser heater system where a $792 \mathrm{~nm}$ Ti-sapphire laser with the pulse length of $10 \mathrm{ps}$ are used to increase the rms energy spread from $2 \mathrm{keV}$ to about $8.4 \mathrm{keV}$. After a total longitudinal compression factor of about 10, the sliced rms energy spread should be about $84 \mathrm{keV}$ at the undulator entrance (at $840 \mathrm{MeV}$ ) in the absence of impedance effects. If one supposes a fundamental Gaussian mode laser with spot much larger than the electron beam size copropagates with a Gaussian electron beam in the laser heater undulator, the energy modulation amplitude is almost the same for all electrons, and the energy profile of heated beam is possibly a saddle distribution, as the black shown in Fig. 1.

\section{A. On HGHG}

Among the various seeding schemes, HGHG is the most compact and pioneering. The high harmonic bunching of HGHG can be described as [29]

$$
b=J_{h}\left(h \Delta \gamma_{s} D\right) \int d p f(p) e^{-i h D \sigma_{E} p},
$$

where $h$ is the harmonic number, $D=k_{s} R_{56} / \gamma, k_{s}$ is the wave number of the seed laser, $R_{56}$ is the strength of the dispersive chicane, $\gamma$ is the electron beam Lorentz factor, 
$\Delta \gamma_{s}$ is the seed laser-induced energy modulation amplitude and $J_{h}$ is the $h$ th order Bessel function, $p=\left(E-E_{0}\right) / \sigma_{E}$ is the dimensionless energy deviation of a particle with an average energy $E_{0}$ and rms energy spread $\sigma_{E}, f(p)$ is the initial longitudinal phase space distribution. For a Gaussian energy distribution, following Eq. (1), the bunching factor can be written as

$$
b_{G}=J_{h}\left(h D \Delta \gamma_{s}\right) \exp \left(-\frac{h^{2} D^{2} \sigma_{E}^{2}}{2}\right) .
$$

For a saddle distribution, which is caused by the energy modulation process in laser heater, using the notations in Ref. [26], i.e., the net longitudinal bunch length compression between the laser heater and the main undulator $C$, the energy modulation induced in the laser heater system $\Delta \gamma_{h}$ and the energy spread at the exit of the photoinjector $\sigma_{H}$, the bunching factor can be written as $[23,26]$

$$
b_{S}=\left|J_{h}\left(h D \Delta \gamma_{s}\right) \exp \left(-\frac{h^{2} \sigma_{H}^{2} C^{2} D^{2}}{2}\right) \times J_{0}\left(h C D \Delta \gamma_{h}\right)\right| .
$$

The predictions made by Eq. (2) have been analyzed intensively in Ref. [24]. The bunching factor draw back fast for a Gaussian energy distribution and this feature limits the feasibility of HGHG at high harmonics. For the nonGaussian case, FERMI's experiment results show an FEL output pulse energy oscillation with the increase of the laser heating [26], which is a meaningful demonstration of Eq. (3).

If we assume a more ideal case that the electron energy is uniformly distributed between $\left[E_{0}-\tau / 2, E_{0}+\tau / 2\right]$, the rms energy spread is then changed to $\sigma_{E}=0.5 \tau / \sqrt{3}$. According to Eq. (1) and the law of Fourier transform for a rectangular pulse [30], the bunching factor for the uniform energy distribution at $h$ th harmonic can be presented as

$$
b_{U}=J_{h}\left(h D \Delta \gamma_{s}\right)|\operatorname{Sinc}(h D \tau / 2)| .
$$

To verify the above-mentioned theoretical predictions and compare different cases, we carry out the single frequency simulations using the universal FEL simulating code GENESIS [31]. In these simulations, we take the main parameters of SXFEL as an example to illustrate the effects of different energy distribution on FEL density modulation process. Considering that the effective energy spread induced by the seed laser in HGHG is limited by the

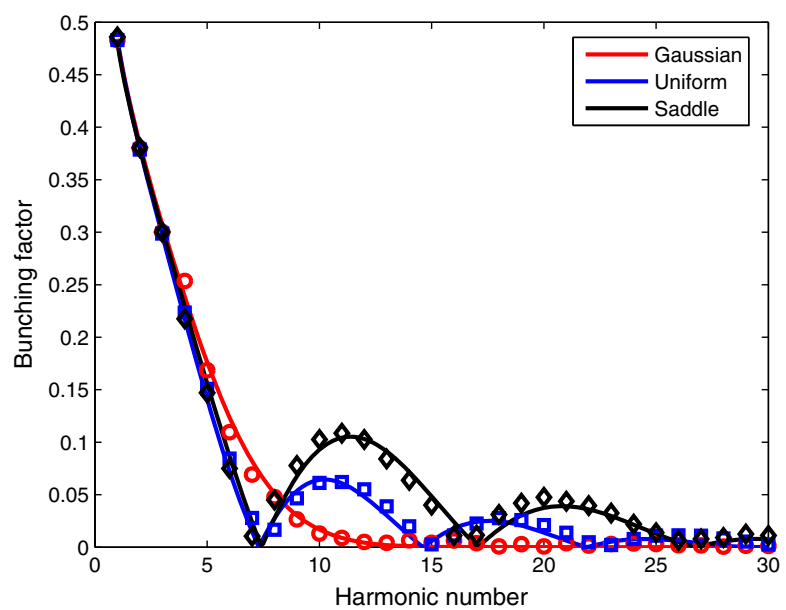

FIG. 2. The evolution of bunching factor with the harmonic number, the red circle is Gaussian results, blue square and the black diamond is for uniform and saddle respectively, the corresponding color line is theoretical derivation of Eqs. (2)-(4).

FEL parameter $\rho$ for the requirement of exponential amplification in the final $8.8 \mathrm{~nm}$ radiator, the energy modulation amplitude $A=\Delta E / \sigma_{E}$ is chosen to be about 5 , and the optimal dispersive strength is chosen to be $k_{1} R_{56} A \approx 1.2$ here.

Figure 2 shows the bunching factor distributions at various harmonic numbers for different cases. The bunching factor oscillations are clearly seen for the uniform and saddle energy spread distribution cases. The amplitudes of the oscillations can be adjusted by setting the energy modulation amplitude and the strength of dispersive chicane. The simulation dots are all at the vicinity of the theoretical value, which is in good agreement with the derivation of Eqs. (2)-(4). This kind of bunching factor oscillation can be used to significantly extend the tuning range of the output wavelength of a single-stage HGHG down to very high harmonics, and makes the generation of soft $x$-ray FEL pulses in a single-stage HGHG possible.

\section{B. On EEHG}

Now we discuss the effects of energy distribution in the EEHG scheme, following similar derivation in Sec. II and the notations in Ref. [11], assuming the frequency of the two seed laser in EEHG is identical, the EEHG bunching factor with typical Gaussian energy distribution can be represented as

$$
b_{G}=\left|\exp \left(-\frac{1}{2}\left[n B_{1}+(m+n) B_{2}\right]^{2}\right) J_{m}\left[-(m+n) A_{2} B_{2}\right] J_{n}\left\{-A_{1}\left[n B_{1}+(m+n) B_{2}\right]\right\}\right| .
$$


$A_{1,2}=\Delta E_{1,2} / \sigma_{E}$ is the beam energy modulation amplitude induced in the modulators. For simplicity, we introduce the $B_{1,2}=R_{56}^{1,2} k \sigma_{E} / E$ for the strengths of the dispersive chicanes, $k$ represents the wave numbers of the seed laser, $m$ and $n$ are integers and the harmonic number of EEHG is $h=m+n$.

For the uniform energy distribution, the exponential term in Gaussian distribution is converted into a Sinc function, and the EEHG bunching factor can be rewritten as

$$
b_{U}=\left|\operatorname{Sinc}\left\{\tau\left[n B_{1}+(m+n) n B_{2} / 2\right]\right\} J_{m}\left[-(m+n) A_{2} B_{2}\right] J_{n}\left\{-A_{1}\left[n B_{1}+(m+n) B_{2}\right]\right\}\right| .
$$

Following the derivation of Eq. (5), the bunching factor with a saddle energy distribution can be presented as

$$
\begin{aligned}
b_{S}= & \mid \exp \left(-\frac{1}{2}\left\{\left[n D_{1}+(m+n) D_{2}\right] C \sigma_{H}\right\}^{2}\right) J_{m}\left[-(m+n) A_{2} B_{2}\right] J_{n}\left\{-A_{1}\left[n B_{1}+(m+n) B_{2}\right]\right\} \\
& \times J_{0}\left\{-C \Delta \gamma_{h}\left[(m+n) D_{2}+n D_{1}\right]\right\} \mid
\end{aligned}
$$

According to the optimal condition of EEHG, the maximum bunching factor and the same sign of $B_{1,2}$ can be attained simultaneously when $n=-1$. Figure 3 shows the simulation results of the bunching factor at different harmonic number. The EEHG parameters used here are $A_{1,2}=2.5$ to obtain a same laser-induced energy spread with HGHG case mentioned above, the wavelength of the two seed lasers are $264 \mathrm{~nm}$ and each harmonic is optimized separately. The optimal relationship between the two dispersive chicanes for EEHG operation is [11]

$$
B_{2}=-\frac{n}{a} B_{1}-\frac{\xi}{a}
$$

where $\xi$ is the solution of $A_{1}\left[J_{n-1}\left(A_{1} \xi\right)-J_{n+1}\left(A_{1} \xi\right)\right]=$ $2 \xi J_{n}\left(A_{1} \xi\right)$.

As mentioned above, the first strong chicane in EEHG will smear out the initial energy spread distribution of the

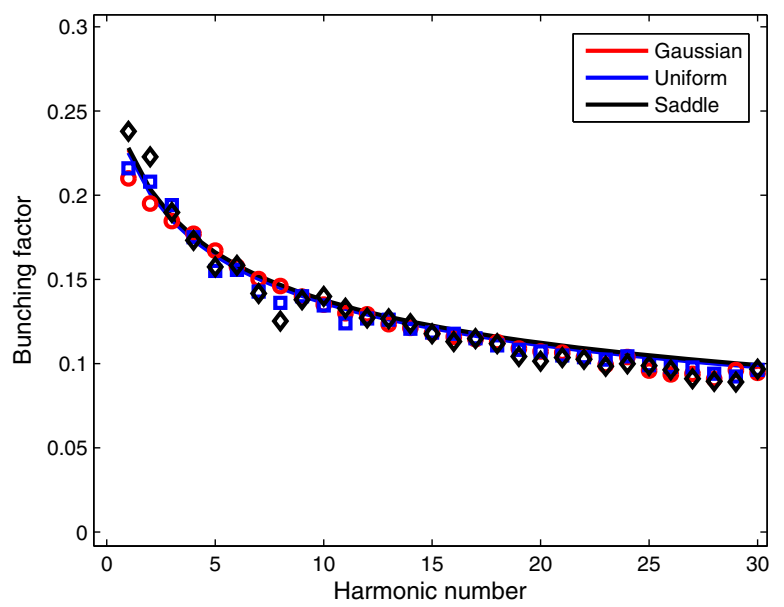

FIG. 3. The EEHG bunching factor vs the harmonic number. The solid curves are calculated from Eqs. (5)-(7), the corresponding colorized dots represent the $3 \mathrm{D}$ simulation results. electron beam, in Fig. 3, the EEHG bunching factor is nearly zero response to the energy distribution and the simulation results are in excellent agreement with the predictions of Eqs. (5)-(7).

\section{On PEHG}

The PEHG scheme [16-18] combines the dogleg and the transverse gradient undulator (TGU) modulator [32], which induce a transverse-longitudinal phase space coupling. When the transversely dispersed electrons pass through the TGU modulator, around the zero crossing of the seed laser, the electrons with the same energy will merge into a same longitudinal phase. PEHG holds the great promise for generating fully coherent short-wavelength radiation. In the frame of 1D theory, the initial beam energy spread is artificially rearranged and fully suppressed in the PEHG scheme by the so-called phase-merging effect. Thus, the bunching factor of PEHG should be independent on the shape of beam energy distributions.

If one takes the transverse effects into accounts, according to Refs. [16-18], the PEHG bunching can be written as

$$
b=J_{h}\left(h D \Delta \gamma_{s}\right) \exp \left(-\frac{h^{2} D \sigma_{x}^{2}}{2 \eta^{2}}\right),
$$

where $\eta$ is the transverse dispersion of the dogleg and $\sigma_{x}$ is the transverse beam size. The reasonable beam size in the TGU modulator is $\sigma_{x}=\sqrt{\epsilon_{x} L_{m} / 2 \gamma}$, with $\epsilon_{x}$ and $L_{m}$ for the normalized horizontal emittance and the modulator length, respectively. It indicates that the bunching of PEHG is the same as the standard HGHG-FEL with an equivalent energy spread of $\sigma_{\text {eff }}=\sigma_{x} / \eta$. PEHG bunching is immune to different energy distributions theoretically.

The PEHG bunching factor evolutions are illustrated in Fig. 4 for the three different energy distribution cases, where the energy modulation amplitude used in PEHG is also $A=5$ to keep the same laser induced energy spread, 


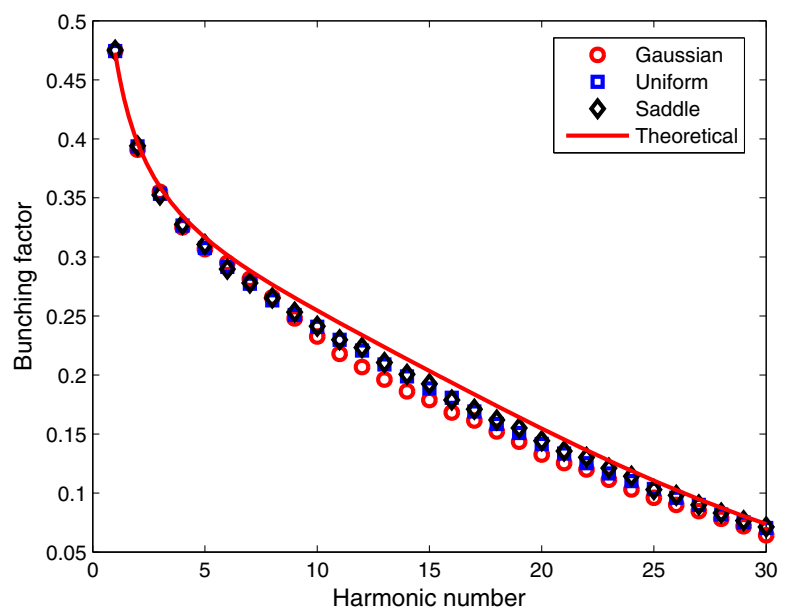

FIG. 4. The evolution of PEHG bunching factor vs the harmonic number, the dots are the simulation results, and the red line is theoretical derivation of the PEHG bunching factor with an effective energy spread.

the length of the modulator is $L_{\mathrm{m}} \approx 1.2$, the dispersion of the dogleg is $\eta=0.85 \mathrm{~m}$ and transverse gradient of TGU is $\alpha=20 \mathrm{~m}^{-1}$. The simulation dots with different energy distribution in PEHG have nearly the same tendency and the red line shows the prediction of Eq. (9) with an effective energy spread.

Considering that the effective energy spread in PEHG comes from the transverse distribution of the electron beam, to be more comprehensive, different transverse beam distribution are also calculated, including Gaussian and uniform. The saddle case is ignored for realistic consideration. The bunching factor obtained for different transverse distributions are summarized in Fig. 5. The bunching factor in PEHG shows an oscillation for the uniform distribution as compared to the Gaussian. It is found that the bunching factor has an increase around the 50th

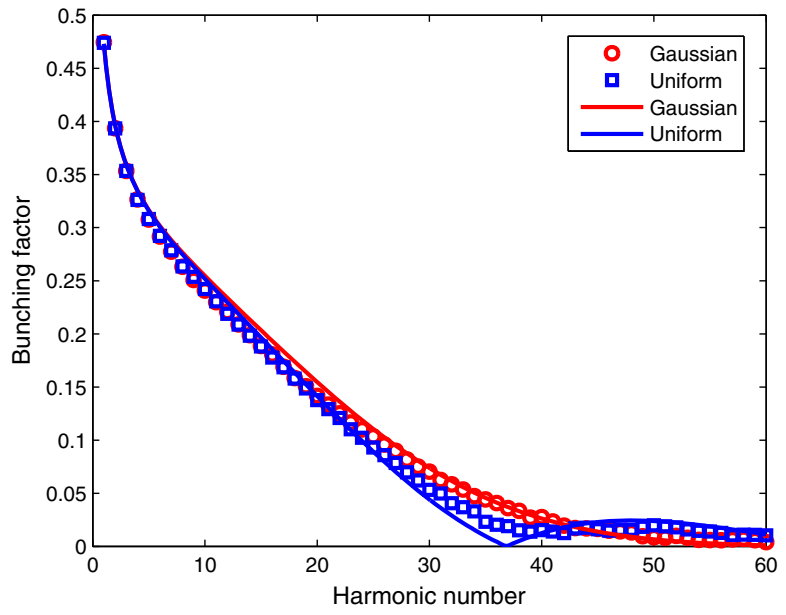

FIG. 5. The PEHG bunching factor vs the harmonic number. The filled curves are calculated by Eqs. (4) and (9), the colorized dots represent the simulation results. harmonic for the uniform energy distribution, which is in good agreement with HGHG theory of Eq. (4) by using an energy spread of $\sigma_{\text {eff }}$. Moreover, the output peak power in tapered FEL can be dramatically improved for uniform transverse beam distribution case by enhancing the optical guiding in the undulator [33]. All these results indicate that the transverse shaping of the electron beam may be an effective way to improve the FEL performance.

\section{OPERATING SXFEL WITH SINGLE-STAGE HGHG}

It has been widely discussed that seeded FELs with total frequency up-conversion factor of 30, e.g., SXFEL, can be realized by EEHG [34] and PEHG [16-18] in a single-stage configuration. In this section, we discuss the feasibility of operating SXFEL with a single-stage HGHG, by properly handling the distribution of the sliced beam energy spread with the laser heater.

It is widely known that the Landau damping of the microbunching instability in the electron beam with a Gaussian energy spread is much more efficient than that with a saddle one. It means that, in order to achieve the same suppression of the microbunching instability, a larger laser energy in the laser heater, or equivalently a larger rms deviation of the electron energy distribution could be needed for the non-Gaussian case. Therefore, to clearly understand and state the tradeoff of using a saddlelike energy distribution instead of the Gaussian one for seeded FELs, start-to-end tracking of the electron beam, including all the components of SXFEL has been carried out. The electron beam dynamics in the photoinjector was simulated with ASTRA [35] to take into account space-charge effects. ELEGANT [36] was then used for the simulation in the remainder of the LINAC. For simplicity, one bunch compressor setup of SXFEL is considered.

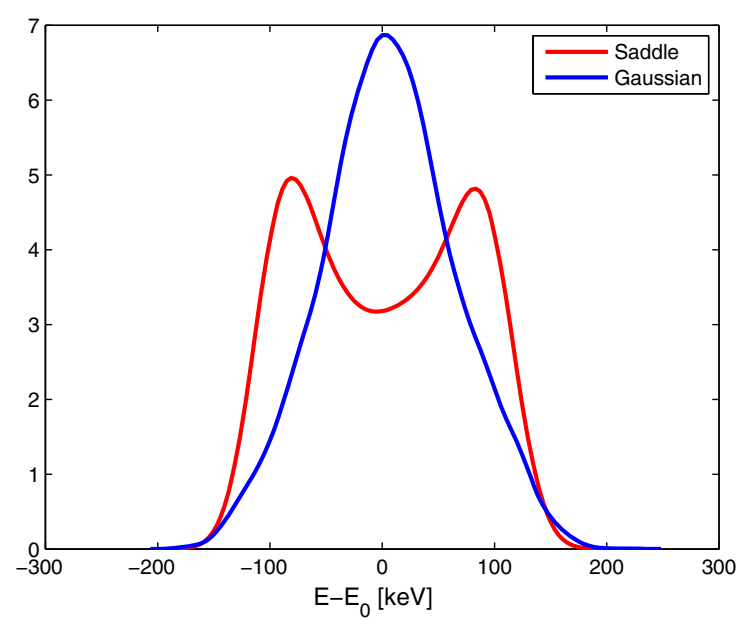

FIG. 6. The saddle and Gaussian sliced beam energy distribution at the exit of the LINAC. 


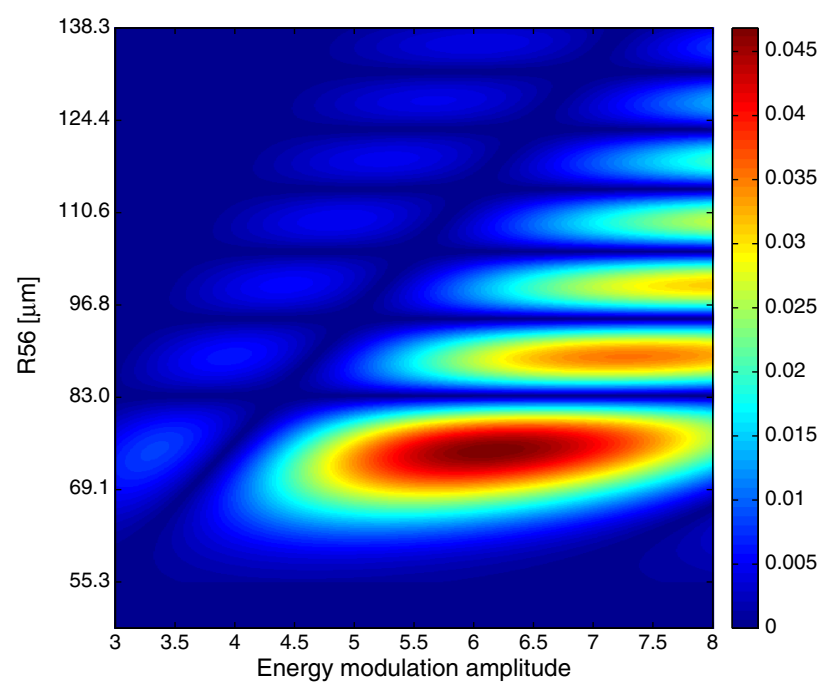

FIG. 7. The 30th harmonic bunching factor in a single-stage HGHG vs the energy modulation amplitude and the strength of the dispersion. A saddlelike energy distribution from ELEGANT with a rms energy spread of $38 \mathrm{keV}$ is used.

In the simulation, the total energy spread of about $20 \mathrm{keV}$ is obtained in the absence of all the impedance effects, i.e., the energy sliced energy spread at the exit of photon injector of $\sim 2 \mathrm{keV}$ and the bunch compression factor of 10 . In further microbunching studies, we first switch off the laser heater, and it is found that the typical sliced beam energy spread is $54 \mathrm{keV}$ at the exit of LINAC. Then two laser-heater cases with the laser size of 0.3 and $1.2 \mathrm{~mm}$ are considered, respectively, while the electron beam size in the heater is $0.3 \mathrm{~mm}$ in both cases. The laser energy is independently optimized to obtain a better microbunching suppression, i.e., a lower sliced beam energy spread here for each case. The energy distribution at the exit of the
LINAC is shown in Fig. 6. According to the simulation, the beam energy distribution shape controlled by the laser heater can be maintained in the LINAC. The optimal energy spread is about 27 and $38 \mathrm{keV}$ for Gaussian and saddle case, which are both better than the case without laser heater. In other words, it results more microbunching and larger energy spread in the saddle case than in the Gaussian one.

According to the previous results, the bunching factor of HGHG can be significantly enhanced with a saddle energy distribution. Using the tracked saddlelike energy distribution, the optimized 30th harmonic bunching factor as a function of the HGHG scheme setup is shown in Fig. 7. One can find that the 30th harmonic bunching factor could be more than $4 \%$ for energy modulation amplitude $A$ around 6, which is strong enough for driving intense coherent radiation at the beginning of the radiator. Moreover, in view of the tradeoff between the seed laser induced energy spread and the available bunching factor, a moderate modulation amplitude of $A=6.5$ is chosen for FEL gain process in the radiator.

In the FEL simulation, the saddle energy distributions from ELEGANT are artificially imported to GENESIS [31] at the entrance of the modulator undulator. The FWHM pulse duration of the $264 \mathrm{~nm}$ seed laser is supposed to be $500 \mathrm{fs}$. In order to fairly compare the HGHG performance for both Gaussian and saddle distributions, the energy spread induced by the seed laser is assumed to be the same in both cases. Figure 8 shows the comparison of output pulse energy along the radiator and the output spectra. The saddle energy spread beam drives a strong coherent radiation at the beginning of the radiator and the saturation length is about $10 \mathrm{~m}$, while the Gaussian one almost starts from shot noise and a much longer radiator is required. After passing through $10 \mathrm{~m}$ long radiator, the relative FWHM bandwidth
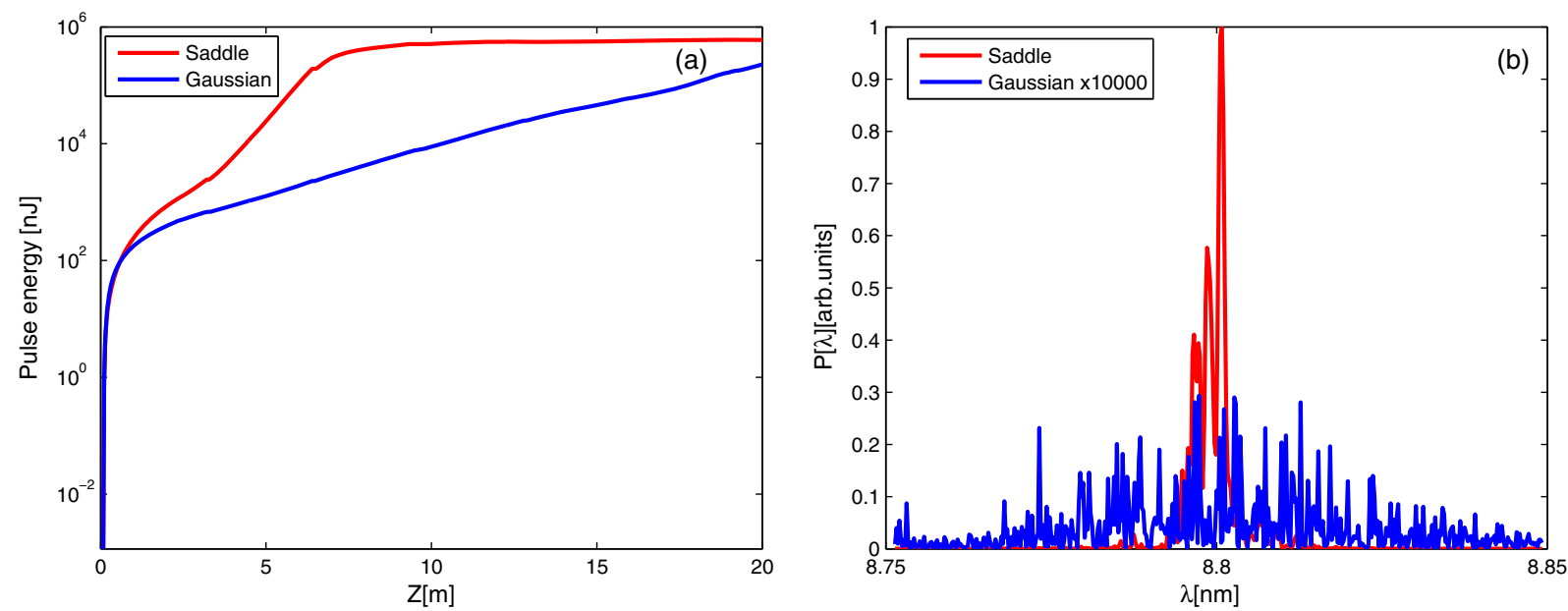

FIG. 8. Comparison of final $8.8 \mathrm{~nm}$ radiation pulse energy (a) and spectra (b) of SXFEL with a single-stage HGHG. The spectra are exported at the undulator position of $10 \mathrm{~m}$, where the saddle distribution is almost saturated, while the Gaussian one is still in exponential gain regime. 
of saddle case is about $0.05 \%$ and 5 times narrower than the Gaussian distribution. The noisy spike and FEL spectrum broaden in the saddle case is induced mainly by the nonlinear energy chirp in the electron beam [37-40]. It is worth stressing that with the recent technology [41-42], the FEL performance can be further improved by removing the beam energy curvature [43].

\section{CONCLUSION}

In this paper, the sliced energy distribution effects on the bunching process in seeded FELs are investigated by using theoretical analysis and numerical simulations. It is found that bunching factors in different seeding mechanisms have quite different dependence on the beam energy distribution. EEHG and PEHG nearly have no response to different energy spread distributions, while a bunching factor oscillation happens in HGHG for uniform and saddle distributions. Moreover, such a bunching factor oscillation in HGHG can be adjusted by setting the energy modulation amplitude and the strength of the dispersive chicane, thus to obtain a large bunching factor at high harmonics.

For the single-stage HGHG operation of a soft x-ray FEL, the start-to-end example in this paper demonstrates that the 30th or even higher harmonic is possible with a moderate energy spread control by using the laser-heater system in the LINAC, even though the saddle distribution has a larger sliced beam energy spread than a Gaussian case. Thus, by manipulating the energy spread distribution, a single-stage HGHG may be used to cover much larger harmonic range than the theoretical predictions under the assumption of Gaussian beam energy spread distribution. However, in order to avoid the temporal coherence degradation due to the nonlinear beam energy curvature, a much shorter seed laser is preferred for high harmonic operation of single-stage HGHG. While in other advanced seeding configurations, i.e., EEHG and PEHG, a long seed lase pulse is allowed to entirely explore the ability of full electron bunch and thus enhance the average FEL brightness.

Finally, it is worth emphasizing that the control of the sliced beam energy spread, both rms deviation and shape is quietly related to many issues, e.g., the required suppression of the microbunching instability, the detailed LINAC setup, and the FEL performances in pursuit. In general, larger laser heater energy, or equivalently a larger rms for the electron energy distribution may be needed in the nonGaussian case. Then for a real FEL machine, except the robust design and self-consistent start-to-end beam tracking, it is likely that the machine flexibility, the accuracy of beam energy spread measurement, the commissioning experiences and efforts will determine the frequency up-conversion limit achievable for different seeded FELs.

\section{ACKNOWLEDGMENTS}

The authors are grateful to Bo Liu and Dao Xiang for helpful discussions. This work was supported by the National Natural Science Foundation of China (21127902, 11175240, 11205234 and 11322550).

[1] L. H. Yu, Phys. Rev. A 44, 5178 (1991).

[2] L. H. Yu et al., Science 289, 932 (2000).

[3] L. H. Yu et al., Phys. Rev. Lett. 91, 074801 (2003).

[4] T. Shaftan and L. H. Yu, Phys. Rev. E 71, 046501 (2005).

[5] E. Allaria et al., Nat. Photonics 6, 699 (2012).

[6] R. Bonifacio et al., Riv. Nuovo Cimento 17, 9 (1990).

[7] J. Wu and L. H. Yu, Nucl. Instrum. Methods Phys. Res., Sect. A 475, 104 (2001).

[8] B. Liu et al., Phys. Rev. ST Accel. Beams 16, 020704 (2013).

[9] E. Allaria et al., Nat. Photonics 7, 913 (2013).

[10] G. Stupakov, Phys. Rev. Lett. 102, 074801 (2009).

[11] D. Xiang and G. Stupakov, Phys. Rev. ST Accel. Beams 12, 030702 (2009).

[12] D. Xiang et al., Phys. Rev. Lett. 102, 074801 (2009).

[13] Z. T. Zhao et al., Nat. Photonics 6, 360 (2012).

[14] D. Xiang et al., Phys. Rev. Lett. 108, 024802 (2012).

[15] E. Hemsing, M. Dunning, C. Hast, T. O. Raubenheimer, S. Weathersby, and D. Xiang, Phys. Rev. ST Accel. Beams 17, 070702 (2014).

[16] H. Deng and C. Feng, Phys. Rev. Lett. 111, 084801 (2013).

[17] C. Feng, H. Deng, D. Wang, and Z. Zhao, New J. Phys. 16, 043021 (2014).

[18] C. Feng, T. Zhang, H. Deng, and Z. Zhao, Phys. Rev. ST Accel. Beams 17, 070701 (2014).

[19] C. Feng, H. X. Deng, M. Zhang, T. Zhang, X. T. Wang, W. Zhang, B. Liu, D. Wang, Z. T. Zhao, in Proceedings of FEL2014, Basel, Switzerland (Shanghai Institute of Applied Physics, CAS, Shanghai, 2014).

[20] Z. T. Zhao, Z. M. Dai, X. F. Zhao, D. K. Liu, Q. G. Zhou, D. H. He, Q. K. Jia, S. Y. Chen, and J.P. Dai, Nucl. Instrum. Methods Phys. Res., Sect. A 528, 591 (2004).

[21] H. Deng et al., Phys. Rev. ST Accel. Beams 17, 020704 (2014).

[22] H. Deng, Nuclear Science and Techniques 25, 010101 (2014).

[23] Z. Huang, M. Borland, P. Emma, J. Wu, C. Limborg, G. Stupakov, and J. Welch, Phys. Rev. ST Accel. Beams 7, 074401 (2004).

[24] Z. Huang et al., Phys. Rev. ST Accel. Beams 13, 020703 (2010).

[25] G. Dattoli et al., arXiv:1410.6614.

[26] E. Ferrari, E. Allaria, W. Fawley, L. Giannessi, Z. Huang, G. Penco, and S. Spampinati, Phys. Rev. Lett. 112, 114802 (2014).

[27] S. Spampinati et al., Phys. Rev. ST Accel. Beams 17, 120705 (2014).

[28] J. Yan, M. Zhang, and H. Deng, Nucl. Instrum. Methods Phys. Res., Sect. A 615, 249 (2010).

[29] K. J. Kim, Z. Huang, and R. Lindberg,Introduction to the physics of Free Electron Laser (2014). 
[30] Bochner and K. Chandrasekharan, Fourier Transforms (Princeton University Press, Princeton, NJ, 1949).

[31] S. Reiche, Nucl. Instrum. Methods Phys. Res., Sect. A 429, 243 (1999).

[32] T. I. Smith, J. M. J. Madey, L. R. Elias, and D. A. G. Deacon, J. Appl. Phys. 50, 4580 (1979).

[33] C. Emma, J. Wu, K. Fang, S. Chen, S. Serkez, and C. Pellegrini, Phys. Rev. ST Accel. Beams 17, 110701 (2014).

[34] D. Xiang and G. Stupakov, Report No. SLAC-PUB-13475, 2008.

[35] K. Flottmann, ASTRA User Manual, http://www.desy.de/ $\sim$ mpyflo.

[36] M. Borland, ANL Advanced Photon Source Report No. LS-287, 2000.
[37] E. L. Saldin, E. A. Schneidmiller, and M. V. Yurkov, Opt. Commun. 202, 169 (2002).

[38] D. Ratner, A. Fry, G. Stupakov, and W. White, Phys. Rev. ST Accel. Beams 15, 030702 (2012).

[39] C. Feng, H. Deng, G. Wang, D. Wang, Z. Zhao, and D. Xiang, Phys. Rev. ST Accel. Beams 16, 060705 (2013).

[40] G. Wang, C. Feng, H. Deng, T. Zhang, and D. Wang, Nucl. Instrum. Methods Phys. Res., Sect. A 753, 56 (2014).

[41] K. Bane and G. Stupakov, Nucl. Instrum. Methods Phys. Res., Sect. A 690, 106 (2012).

[42] P. Emma et al., Phys. Rev. Lett. 112, 034801 (2014).

[43] H. Deng et al., Phys. Rev. Lett. 113, 254802 (2014). 